\title{
Centralized normalization of harmonic voltages in the supply network for traction substations
}

\author{
L.I.Kovernikova \\ The Siberia Branch of the Russian Academy of Sciences \\ Energy Systems Institute \\ 130, Lermontov Str., 664033 Irkutsk (Russia) \\ Phone:+7 3952 426495, fax: +7 3952 426796, e-mail: kovernikova@isem.sei.irk.ru
}

\begin{abstract}
The paper solves the problem of centralized reduction of harmonic voltages at the nodes connecting traction substations on railway to the supply HV network via the passive C-type filters. The results of measurements at the connection nodes of traction substations to the supply network of the voltage quality indices that are due to non-sinusoidality are presented. Changes of the indices at different configurations of the supply network are analyzed. Mathematical expressions to determine parameters of the C-type filters, the algorithms for selecting network nodes where they will be installed are given. The parameters of the selected nodes for $220 \mathrm{kV}$ network supplying traction substations with power and also the estimates of the efficiency of work of these filters for different network configurations are presented as an example. The active power losses in the selected filters are calculated.
\end{abstract}

\section{Key words}

Harmonic measurement, harmonic distortion, harmonic limits, centralized normalization, filters design.

\section{Introduction}

Traction substations of the railway are supplied with power from the $110-220 \mathrm{kV}$ AC public network at a frequency of $50 \mathrm{~Hz}$. Two triple-wound transformers with a capacity of 40 MVA each are installed at the traction substations. Traction load is covered from the winding of the average voltage $27.5 \mathrm{kV}$. The rest of load is covered from the winding of low voltage 6.6 (11) $\mathrm{kV}$. The traction load is distorting. The DC motor of the electric locomotive is a nonlinear device of the traction load. The motor is supplied with power through the single-phase two-pulse rectification circuit. The non-sinusoidal current consumed by the motor flows in the HV network and creates non-sinusoidal voltage drops. As a result, the voltage curve shape at the point of traction substation connection to the supply network is distorted. Voltage distortions exceed the requirements established by the Russian standard [1].

In East Siberia the railway and electric network supplying it are very long. The traction substations are installed at a distance of 40-60 $\mathrm{km}$. Each section of the railway between two substations is supplied with power from two sides. However, sometimes, for example, during maintenance, such section is supplied from one traction substation. The interval between trains is from 5 to 20 minutes.

With respect to the supply network the traction substations can be considered as distributed loads. It seems that it would be economically sound to reduce voltage distortions on the long section of supply network in a centralized way. The problem of centralized harmonic voltage reduction in an electric network has already been treated in publications $[1,2]$. In [1] it is noted that for some cases the lower cost and smaller required areas make it possible to consider the centralized system of reducing harmonic voltages, even if it is less effective for some harmonics. In [2] consideration is given to the centralized reduction of harmonic voltages in the radial distribution $12-23 \mathrm{kV}$ network with many distributed harmonic sources of low power. Placement of sources and the values of harmonic currents are unknown. Harmonic voltages can be reduced by using passive filters.

In the suggested work the problem of centralized reduction of harmonic voltages at the nodes of the longdistance HV network with distributed nonlinear load is solved on the example of network supplying traction substations with power. 


\section{Description of the considered network}

The possibility for centralized reduction of harmonic voltages at the nodes of long-distance network with distributed nonlinear load is studied on the section of real $220 \mathrm{kV}$ network about $900 \mathrm{~km}$ long. The network supplies 23 traction substations with power. The operating parameters and the indices characterizing the extent of harmonic distortion: the $n$-th harmonic factor $\left(\boldsymbol{K}_{\boldsymbol{U}(\boldsymbol{n})}\right)$ and the total harmonic distortion $\left(\boldsymbol{K}_{\boldsymbol{U}}\right)$ were measured at seven traction substations [1]. The results of measurements have showed that the average power of traction load was 4-8 MW and the maximum one reached 15-20 MW. The analysis of measured currents has revealed prevalence of currents of the $3 \mathrm{rd}$, 5th and 7 th harmonics in them. The 3rd harmonic current makes up $25-30 \%$, the 5 th - about $10 \%$, the 7 th - some $8 \%$. Measurements of the indices $\boldsymbol{K}_{\boldsymbol{U}(\boldsymbol{n})}$ and $\boldsymbol{K}_{\boldsymbol{U}}$ are presented in Table 1. The same Table gives the normally admissible values of $\boldsymbol{K}_{\boldsymbol{U}(\boldsymbol{n})}$ and $\boldsymbol{K}_{\boldsymbol{U}}$ determined in [1]. The measured values of $\boldsymbol{K}_{\boldsymbol{U}(\boldsymbol{n})}$ and $\boldsymbol{K}_{\boldsymbol{U}}$ that exceed the normally admisible values are given in bold type. The admissible values of $\boldsymbol{K}_{\boldsymbol{U}(\boldsymbol{n})}$ and $\boldsymbol{K}_{\boldsymbol{U}}$ are exceeded at four substations.

TABLE 1. - Measured $\boldsymbol{K}_{\boldsymbol{U}(\boldsymbol{n})}$ and $\boldsymbol{K}_{\boldsymbol{U}}(\%)$

\begin{tabular}{|l|c|c|c|c|}
\hline $\begin{array}{l}\text { Substa- } \\
\text { tion }\end{array}$ & $\boldsymbol{K}_{\boldsymbol{U}}$ & $\boldsymbol{K}_{\boldsymbol{U}(3)}$ & $\boldsymbol{K}_{\boldsymbol{U}(5)}$ & $\boldsymbol{K}_{\boldsymbol{U}(7)}$ \\
\hline S1 & 1.99 & 0.76 & 1.34 & 0.92 \\
\hline S2 & 1.92 & 0.84 & 1.13 & 0.81 \\
\hline S3 & 1.91 & 0.89 & 1.02 & 0.70 \\
\hline S6 & $\mathbf{2 . 5 4}$ & 1.00 & $\mathbf{2 . 3 2}$ & 0.76 \\
\hline S12 & $\mathbf{2 . 0 1}$ & $\mathbf{1 . 7 1}$ & 1.22 & 0.69 \\
\hline S13 & $\mathbf{2 . 8 6}$ & $\mathbf{2 . 0 0}$ & $\mathbf{2 . 0 7}$ & 0.82 \\
\hline S14 & $\mathbf{3 . 0 9}$ & $\mathbf{2 . 0 6}$ & $\mathbf{2 . 2 7}$ & 0.96 \\
\hline Norms & 2.0 & 1.5 & 1.5 & 1.0 \\
\hline
\end{tabular}

The values of $\boldsymbol{K}_{\boldsymbol{U}(\boldsymbol{n})}$ and $\boldsymbol{K}_{\boldsymbol{U}}$ at the connection nodes of other traction substationsB were estimated by using the software package HARMONICS. The initial information was represented in the calculations by the measurements of harmonic currents and voltages, information on the network configuration and parameters of its elements, load structure and amount. The models of traction loads were constructed on the basis of measurements of harmonic current components. The measured $\boldsymbol{K}_{\boldsymbol{U}(\boldsymbol{n})}$ and $\boldsymbol{K}_{\boldsymbol{U}} \quad$ were applied for verification of the network condition. Fig. 1 presents $\boldsymbol{K}_{\boldsymbol{U}(\boldsymbol{n})}$ and $\boldsymbol{K}_{\boldsymbol{U}}$ calculated for all 23 nodes for the network configuration, in which each section of the traction network is supplied with power from two substations. We will call this condition normal. The curves show that the admissible values of $\boldsymbol{K}_{\boldsymbol{U}(3)}$ are exceeded at $8, \boldsymbol{K}_{\boldsymbol{U}(5)}$ - at $12, \boldsymbol{K}_{\boldsymbol{U}(7)}{ }^{-}$at $4, \boldsymbol{K}_{\boldsymbol{U}}$ - at 13 substations.

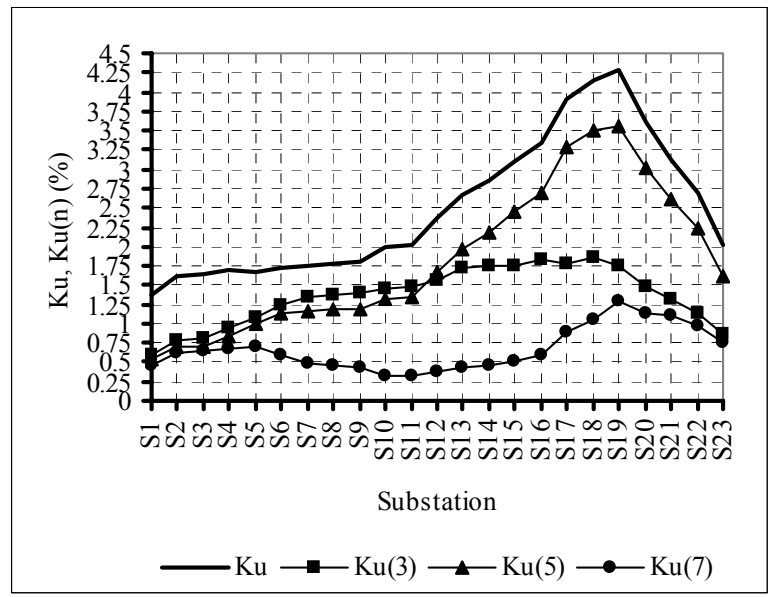

Fig. 1. $\boldsymbol{K}_{\boldsymbol{U}(\boldsymbol{n})}$ and $\boldsymbol{K}_{\boldsymbol{U}}$ at the connection nodes of traction substations.

When the network configuration is changed, for example because of line tripping, the values of harmonic voltages at the nodes can change, since resistances of paths, on which currents flow, vary and therefore, currents are redistributed. The impact of network changes on $\boldsymbol{K}_{\boldsymbol{U}(3)}$, $K_{U(5)}, \quad K_{U(7)}$ was studied by calculation of the $3 \mathrm{rd}$, 5th, 7 th harmonic conditions for 18 network configurations. Figs. 2-4 illustrate the calculation results of $\boldsymbol{K}_{\boldsymbol{U}(3)}, \boldsymbol{K}_{\boldsymbol{U}(5)}$, $\boldsymbol{K}_{\boldsymbol{U}(7)}$ at the network nodes, when the lines supplying traction substations with power were tripped. The railway sections between two traction substations were supplied with power from one substation. The normal condition in the Figures is represented by the curve with large boxes. The Figures show that in the majority of conditions the values of indices differ insignificantly from the indices for normal condition. In several conditions the values of indices increase noticeably. In such a case the filters can be chosen for the normal network condition and the filter efficiency can be tested for the rest of conditions.

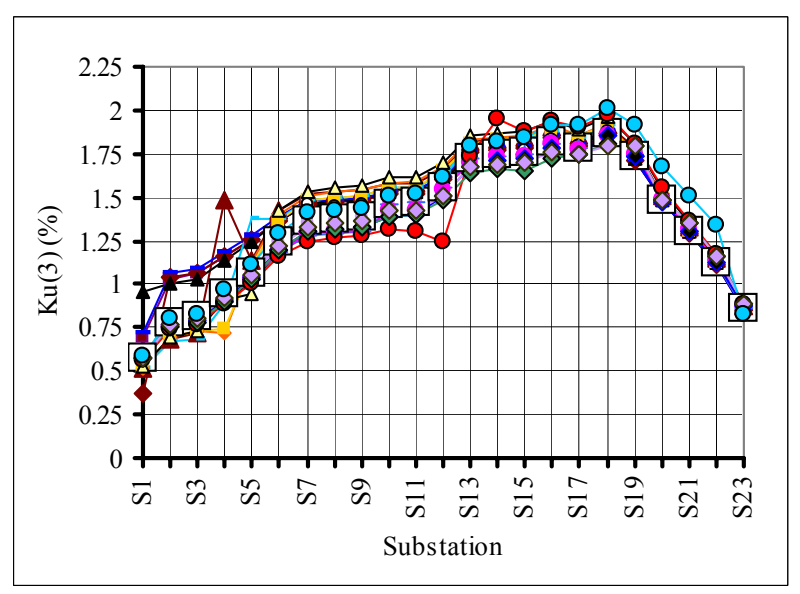

Fig. 2. The values of $\boldsymbol{K}_{\boldsymbol{U}(3)}$ for different configurations of the supply network 


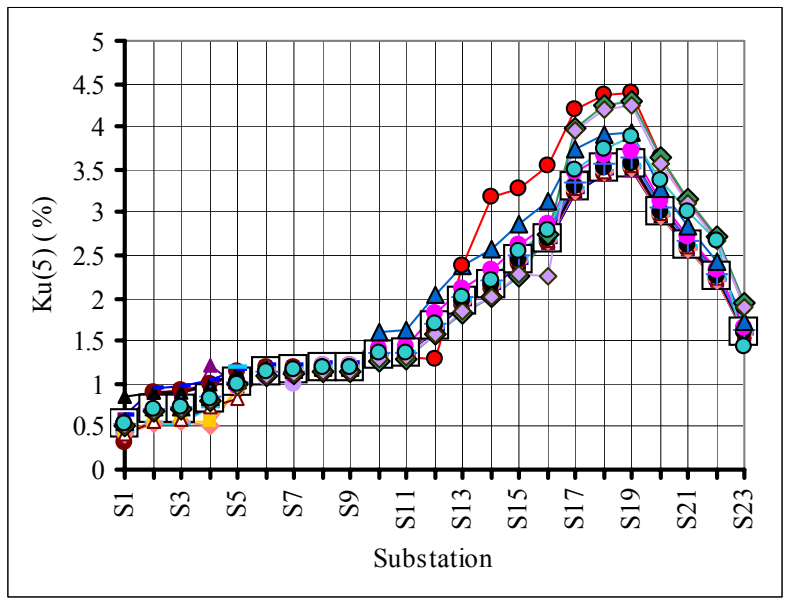

Fig. 3. The values of $\boldsymbol{K}_{\boldsymbol{U}(5)}$ for different configurations of the supply network

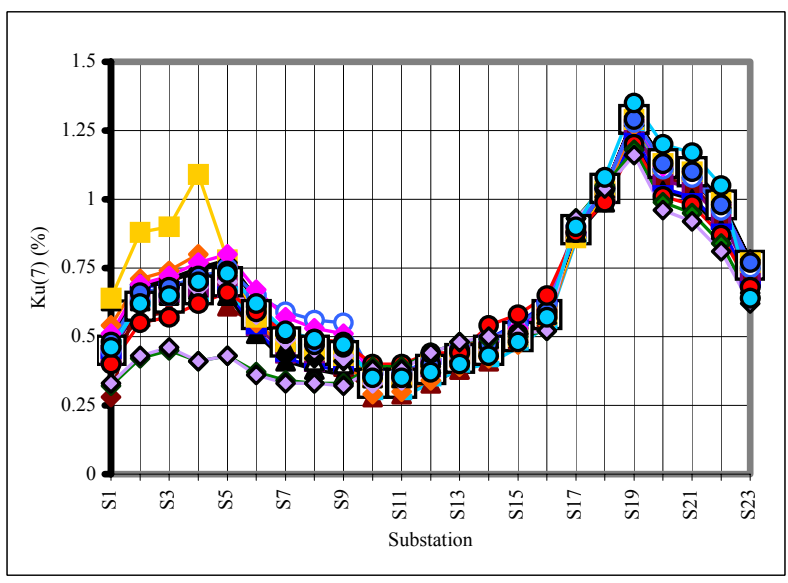

Fig. 4. The values of $\boldsymbol{K}_{\boldsymbol{U}(7)}$ for different configurations of the supply network

\section{Determination of filter parameters}

The C-type filter is chosen for centralized normalization of harmonic voltages at the network nodes. The filter has low losses at the fundamental frequency. The filter parametrs should be chosen so that the filter provided desirable nodal voltage at the harmonic $U_{n F}$ or the desirable value of the index that will be denoted by $\boldsymbol{K}_{\boldsymbol{U}(\boldsymbol{n}) \boldsymbol{F}}$. The value equal to $(0.5-0.75)$ of $\boldsymbol{K}_{\boldsymbol{U}(\boldsymbol{n}) N}$ can be taken as the desirable voltage value $\boldsymbol{K}_{U(\boldsymbol{n}) \boldsymbol{F}} \cdot \boldsymbol{K}_{\boldsymbol{U}(\boldsymbol{n}) N}$ is a norm for the index $\boldsymbol{K}_{\boldsymbol{U}(\boldsymbol{n})}$ [1]. If with respect to some node the electric network and load at the $\boldsymbol{n}$-th harmonic are represented as current sources with admittances, the nodal voltage $\boldsymbol{U}_{n}$ will be determined from the expression

$$
\dot{U}_{n}=\frac{\dot{I}_{n S}+\dot{I}_{n L}}{Y_{n S}+Y_{n L}},
$$

where $\dot{I}_{n s}, \dot{I}_{n L}$ - currents of the current sources of the network and load, $\boldsymbol{Y}_{n s}=\boldsymbol{g}_{n s}+\boldsymbol{j} \boldsymbol{b}_{n s}, \quad \boldsymbol{Y}_{n L}=\boldsymbol{g}_{n L}+\boldsymbol{j} \boldsymbol{b}_{n L}$ admittances of current sources of the network and load.
The voltage can be reduced by increasing the denominator, what in turn can be achieved by the filter admittance $\boldsymbol{Y}_{\boldsymbol{n} \boldsymbol{F}}$

$$
\dot{U}_{n F}=\frac{\dot{I}_{n S}+\dot{I}_{n L}}{Y_{n S}+Y_{n L}+Y_{n F}} .
$$

In [6] it is suggested the use of conductance as such an admittance. Then the filter for the $\boldsymbol{n}$-th harmonic should have the value of the input resistance $\boldsymbol{R}_{\boldsymbol{F}}$ that will provide decrease of the $\boldsymbol{n}$-th harmonic voltage. The resistance value $\boldsymbol{R}_{\boldsymbol{F}}$ can be determined accurately enough, when expressions (1) and (2) are applied jointly

$$
\boldsymbol{R}_{F}=\frac{\boldsymbol{K}_{U(n) F}}{\left(\boldsymbol{K}_{U(n)}-\boldsymbol{K}_{U(n) F}\right) \sqrt{\left(\boldsymbol{g}_{n S}+\boldsymbol{g}_{n L}\right)^{2}+\left(\boldsymbol{b}_{n S}+\boldsymbol{b}_{n L}\right)^{2}}},
$$

where $\boldsymbol{K}_{\boldsymbol{U}(\boldsymbol{n})}, \boldsymbol{K}_{\boldsymbol{U}(\boldsymbol{n}) \boldsymbol{F}^{-}}$- values of indices at the node without the filter and with it.

Schematic diagram of the C-type filter is given in Fig. 5. The following filter parameters must be determined: $\boldsymbol{X}_{C 1}, \boldsymbol{X}_{C 2}, \boldsymbol{X}_{L}, \boldsymbol{R}_{L}, \boldsymbol{R}$ that are calculated for the fundamental frequency.

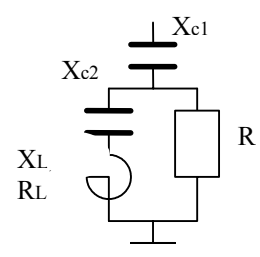

Fig. 5. Schematic diagram of the filter

At the fundamental frequency the filter is a reactive power source to maintain the voltage value $\boldsymbol{U}_{1}$. The reactive power value $\boldsymbol{Q}_{C 1}$ can be determined by calculating the condition at the fundamental frequency or by using the annual curves of reactive power consumed by load. At the adjusted $\boldsymbol{n}$-th harmonic the filter decreases the $\boldsymbol{n}$-th harmonic voltage value. Resistances of the filter condensers are neglected. The filter parameters are determined, provided the following conditions are met:

1) at the fundamental frequency the filter generates reactive power $\boldsymbol{Q}_{C 1}$,

2) at the fundamental frequency to decrease the power losses $\boldsymbol{X}_{\boldsymbol{L}}=-\boldsymbol{X}_{\boldsymbol{C} 2}$,

3) the $\boldsymbol{n}$-th harmonic input reactance of the filter is equal to zero, input resistance of the filter equals $\boldsymbol{R}_{\boldsymbol{F}}$.

Additionally the quality factor of reactor, i.e. the value of ratio $\frac{X_{L}}{R_{L}}$ is given.

In order to calculate filter parameters we use the expression for input impedance of the $\boldsymbol{n}$-th harmonic

$$
Z_{n F}=\frac{\left(R_{L}+j X_{n L}-j X_{n C 2}\right) R}{\left(R_{L}+R\right)+j\left(X_{n L}-X_{n C 2}\right)}-j X_{n C 1},
$$


where $\boldsymbol{X}_{n L}=n \boldsymbol{X}_{L}, \boldsymbol{X}_{n C 1}=\frac{\boldsymbol{X}_{C 1}}{\boldsymbol{n}}, \boldsymbol{X}_{n C 2}=\frac{\boldsymbol{X}_{\boldsymbol{C} 2}}{\boldsymbol{n}}$.

The values of active resistances of the $\boldsymbol{n}$-th harmonic are taken equal to the values of resitances at fundamental frequency. Thus, the filter parameters can be determined from the following expressions:

$\boldsymbol{X}_{\boldsymbol{C} 1}=\sqrt{3} \boldsymbol{U}_{1} / \boldsymbol{Q}_{\boldsymbol{C} 1}$,

$\boldsymbol{X}_{\boldsymbol{L}}=\left(\boldsymbol{k}^{2}+(\boldsymbol{B}+\boldsymbol{q})^{2}\right) \boldsymbol{X}_{C l} /\left(\boldsymbol{B}^{2} \boldsymbol{k n}\right)$,

$\boldsymbol{X}_{C 2}=-\boldsymbol{X}_{L}$,

$\boldsymbol{R}_{L}=\left(\boldsymbol{k}^{2}+(\boldsymbol{B}+\boldsymbol{q})^{2}\right) \boldsymbol{X}_{\boldsymbol{C} 1} \boldsymbol{q} /\left(\boldsymbol{B}^{2} \boldsymbol{k n}\right)$,

$\boldsymbol{R}=\boldsymbol{X}_{L} \boldsymbol{B}$,

where $\boldsymbol{U}_{1}$ - the value of fundamental frequency voltage at the node of filter connection, $\quad k=\left(\boldsymbol{n}^{2}-1\right) / \boldsymbol{n}$, $\boldsymbol{q}=\boldsymbol{R}_{L} / \boldsymbol{X}_{L}, \quad \boldsymbol{B}=\left(\boldsymbol{k}^{2}+\boldsymbol{q}^{2}\right) \boldsymbol{X}_{C 1} /\left(\boldsymbol{R}_{\boldsymbol{F}} \boldsymbol{k n}-\boldsymbol{q} \boldsymbol{X}_{C 1}\right)$.

\section{Selection of filters}

The filters are selected starting with the number of the lowest order harmonic. For any network with several nonlinear loads several options of filters of the n-th harmonic can be selected both in terms of quantity and allocation of filters in the network. Each node in the network has specific features that are characterized by network parameters and condition which will influence the parameters of filters. Normally the filters are selected on the basis of multivariant calculations. While choosing the option it is neessary to obtain the lowest quantity of filters and the lowest number of nodes to be used for installation of filters. It is necessary to give preference to the options in which:

1) filters extend their action to a larger number of neighboring nodes,

2) filters decrease harmonic voltage by a large value,

3) filters have the least active power losses.

Selection of filters should be started with selection of design network configuration and condition.

\section{A. Selection of network configurations and load conditions.}

For this purpose we analyze the most probable network configurations including maintenance ones and the conditions of maximum and minimum loads, i.e. the conditions with connected and disconnected condensers for compensation of reactive power, etc. For the selected network configuraitons and load conditions we calculate the indices $\boldsymbol{K}_{\boldsymbol{U}(\boldsymbol{n})}$ for all the harmonics, for which the standard requirements were not met [1]. Based on the analysis of the calculated $\boldsymbol{K}_{\boldsymbol{U}(\boldsymbol{n})}$ we select the network configuration and load conditions for which the values of the indices exceed $\boldsymbol{K}_{\boldsymbol{U}(\boldsymbol{n}) \boldsymbol{N}}$ at the largest number of nodes for the largest number of harmonics. This network configuration and load conditions are taken as design ones. After calculations of $\boldsymbol{K}_{\boldsymbol{U}(\boldsymbol{n})}$ in the design condition for each harmonic we form the groups of nodes at which the values of $\boldsymbol{K}_{\boldsymbol{U}(\boldsymbol{n}) \boldsymbol{N}}$ are exceeded and which will be considered as potential nodes for installation of filters.
For the design configuration and load conditions we can take an ordinary operating network configuration and ordinary load conditions. For example the results of $\boldsymbol{K}_{\boldsymbol{U}(\boldsymbol{n})}$ calculations that are presented in Figures $2-4$ show that for the considered network normal condition can be taken for the design network configuration and load condtions.

\section{B. Choice of nodes for installation of filters.}

Choice of nodes for installation of filters under centralized normalization of harmonic voltages is the most challenging task. Two approaches are suggested to select the nodes.

1) The first approach. For each node of a formed set we successively calculate filter parameters. Then we calculate harmonic conditions of the network with each filter, the indices $\boldsymbol{K}_{\boldsymbol{U}(\boldsymbol{n})}$ at nodes and power losses in a filter. Further we estimate an impact of the filter on the value $\boldsymbol{K}_{\boldsymbol{U}(\boldsymbol{n})}$ at other nodes of the considered network and analyze the results obtained. Based on the analysis we choose the node at which filter installation will give the most efficient solution. If for the considered harmonic one filter is not sufficient to decrease the value $\boldsymbol{K}_{\boldsymbol{U}(\boldsymbol{n})}$ at all nodes, we choose the second node for filter installation and so on. After selection of one or several filters whose installation will decrease the values of $\boldsymbol{K}_{\boldsymbol{U}(\boldsymbol{n}) \boldsymbol{F}}$, the calculation for the given harmonic ends. Then in the same manner nodes and filters are selected for the next harmonic. For the initial network condition we assume the condition with filters for the previous harmonic.

2) The second approach. Test filter is suggested to choose the nodes where the filters should be installed. The idea of test filter was suggested in [7]. Test filter is a filter with a resistance of $1 \mathrm{Ohm}, 10 \mathrm{Ohm}, 100 \mathrm{Ohm}$, etc. It is installed at each node planned for filter installation, network condition is calculated for the analyzed harmonic and filter efficiency is estimated in terms of harmonic voltage decrease throughout the entire considered network. For the analyzed network the test filter with the input resistance of $1 \mathrm{Ohm}$ has proved to be efficient for searching of the nodes to install the filteres of the 3rd and 5th harmonics, whereas the test filter with the resistance of $100 \mathrm{Ohm}$ is efficient for detecting the nodes to install the filter of the 7 th harmonic. Figure 6 illustrates test filter application for the search of node to accommodate filter of the 3rd harmonic. The test filter with a resistance of $1 \mathrm{Ohm}$ was placed at the nodes denoted by S11-S20. For each case we calculated the index $\boldsymbol{K}_{\boldsymbol{U}(3)}$ at all the nodes in the considered network. The curves show that the filter installed at node $\mathrm{S} 17$ gives the best results in terms of decrease in $\boldsymbol{K}_{\boldsymbol{U}(3)}$ at the nodes. 


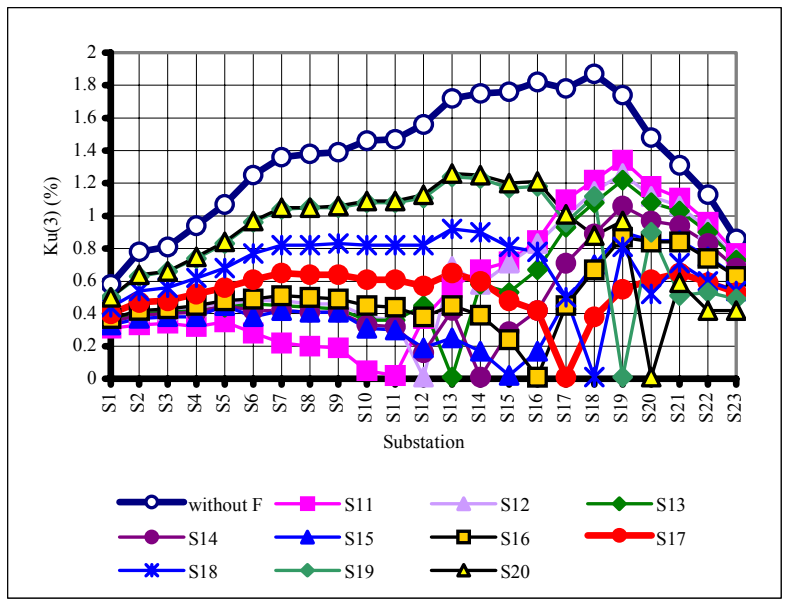

Fig.6. Changes in $\boldsymbol{K}_{\boldsymbol{U}(3)}$ at nodes of the network with test filter

\section{An example of chosen filters}

The example of filters chosen for the $3^{\text {rd }}, 5^{\text {th }}$ and $7^{\text {th }}$ harmonics is presented for the considered network. Based on the measurements (Table 1) and calculations (Fig.1) it is necessary to reduce the values of indices $\boldsymbol{K}_{\boldsymbol{U}(3)}$, $\boldsymbol{K}_{U(5)}, \quad \boldsymbol{K}_{\boldsymbol{U}(7)}$ in the considered network. To do so it is necessary to place filters of the $3^{\text {rd }}, 5^{\text {th }}$ and $7^{\text {th }}$ harmonics. The calculations show that it suffices to accommodate the filters of the $3^{\text {rd }}, 5^{\text {th }}$ and $7^{\text {th }}$ harmonics on the $220 \mathrm{kV}$ buses of substation S17 and then the values of the indices will not exceed the standards established in [1]. Figure 7 presents the curves for $\boldsymbol{K}_{U(\boldsymbol{n})}$ at nodes in the considered $220 \mathrm{kV}$ network with filters for the design condition. Parameters of the chosen filters are presented in Table 2.

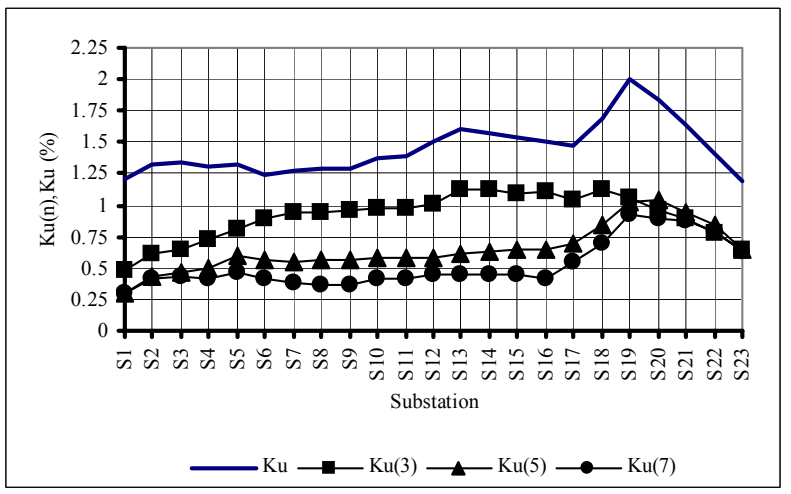

Fig. 7. $\boldsymbol{K}_{\boldsymbol{U}(\boldsymbol{n})}$ at the network nodes with filters

\section{Efficiency estimation of the chosen filters}

Eighteen special calculations were made to estimate the efficiency of filters of the $3^{\text {rd }}, 5^{\text {th }}$ and $7^{\text {th }}$ harmonics that were accommodated on the $220 \mathrm{kV}$ buses of substation S17. In each of the calculations network configuration was changed by disconnecting the line feeding one of the traction substations and the values of indices $\boldsymbol{K}_{\boldsymbol{U}(3)}$, $\boldsymbol{K}_{U(5)}, \quad \boldsymbol{K}_{U(7)}$ at the nodes throughout the network were calculated. The results of the calculations are presented in Figs. 8-10. For harmonics 3 and 5 only in 2 of 18 conditions the indices $\boldsymbol{K}_{U(3)}$ and $\boldsymbol{K}_{\boldsymbol{U}(5)}$ did not decrease, for harmonic 7 one more condition was added to the previous two. On the whole the result obtained is rather good. It shows the possibility to centrally decrease harmonic voltages at nodes in the network that feeds traction substations.

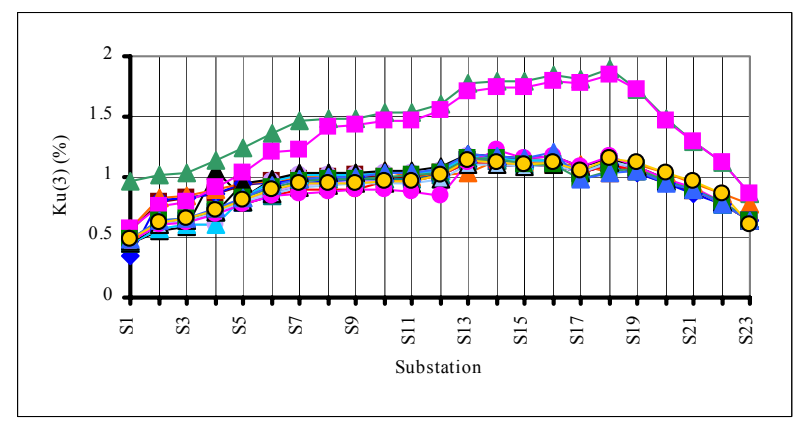

Fig. 8. $\boldsymbol{K}_{U(3)}$ at the network nodes with filters

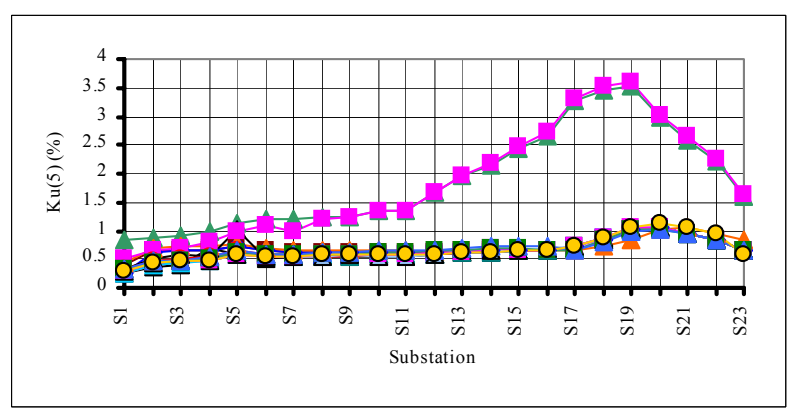

Fig. 9. $\boldsymbol{K}_{\boldsymbol{U}(5)}$ at the network nodes with filters

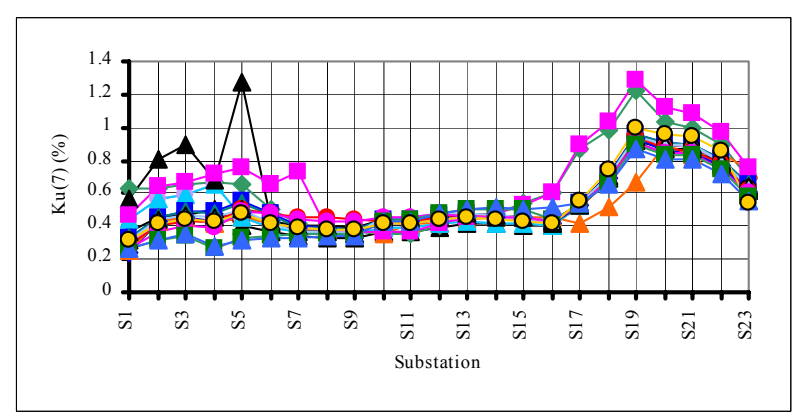

Fig. 10. $\boldsymbol{K}_{\boldsymbol{U}(7)}$ at the network nodes with filters

TABLE 2. - Parameters of filters

\begin{tabular}{|l|c|c|c|}
\hline \multirow{2}{*}{ Parameter } & \multicolumn{3}{|c|}{ Harmonic } \\
\cline { 2 - 4 } & 3 & 5 & 7 \\
\hline $\mathrm{Q}_{\mathrm{C} 1}, \mathrm{MBAp}$ & 4. & 3. & 3. \\
\hline $\mathrm{X}_{\mathrm{Cl}}, \mathrm{OM}_{\mathrm{y}}$ & 12100. & 16133. & 16133. \\
\hline $\mathrm{X}_{\mathrm{L}}, \mathrm{OM}_{\mathrm{y}}$ & 1514. & 673. & 337. \\
\hline $\mathrm{R}_{\mathrm{L}}, \mathrm{OM}_{\mathrm{yyy}}$ & 25. & 11. & 6. \\
\hline $\mathrm{X}_{\mathrm{C} 2}, \mathrm{OM}_{\mathrm{y}}$ & 1514. & 673. & 337. \\
\hline $\mathrm{R}, \mathrm{OM}$ & 170200. & 182070. & 40954. \\
\hline
\end{tabular}




\section{E. Analysis of active power losses in filters}

Active power losses for the fundamental frequency and the frequency of the tuned harmonic were calculated for the chosen filters and presented in Table 3. For comparison - the losses of idle active power of one transformer at traction substation make up $54 \mathrm{~kW}$, the short circuit losses - $220 \mathrm{~kW}$. Harmonic voltages were reduced by three filters at 13 substations that accommodate 26 transformers.

TABLE 3. - Active power losses in filters

\begin{tabular}{|l|c|c|c|}
\hline \multirow{2}{*}{ Parameter } & \multicolumn{3}{|c|}{ Harmonic } \\
\cline { 2 - 4 } & 3 & 5 & 7 \\
\hline $\mathrm{P}(1), \mathrm{kW}$ & 34. & 30. & 12. \\
\hline $\mathrm{P}(\mathrm{n}), \mathrm{kW}$ & 14. & 4. & 2. \\
\hline
\end{tabular}

\section{Conclusions}

1. The possibility is shown to centrally normalize harmonic voltages with the passive filters of C-type at the nodes of an extended high voltage network feeding traction substation.

2. The mathematical expressions are derived to determine the parameters of the C-type filters.

3. The algorithms are developed to search for the network nodes to be used for installation of filters.

4. The example of filters of the $3^{\text {rd }}, 5^{\text {th }}$ and $7^{\text {th }}$ harmonics is given for the extended $220 \mathrm{kV}$ network that feeds traction substations. Their efficiency is estimated for different network configurations.

5. Active power losses in filters are estimated.

\section{Acknowledgement}

The work was supported by the grant of the Leading Scientific School of the RF HШ-1857.2008.8

\section{References}

[1] State Standard 13109-97. Power quality. Electromagnetic compatibility of technical equipment. Power quality limits in electrical systems. - M., 1998. (Russia)

[2] Diakov A.F., Ishkin V.Kh. X., Mamikonyants L.G. Urgent problems in electric power industry// Elektrichestvo, №6, 1997. p.61-68. (Russia)

[3] Thomas H. Ortmeyer, Takashi Hiyama. Distribution system harmonic filter planning. IEEE Transaction on Power Delivery, vol.11, №4, October 1996

[4] Czarnecki L.S. An overview of methods of harmonic suppression in distribution systems. In IEEE Summer Meeting, 2000, Seattle, Washington, USA.

[5] Arrillaga, Jos. Power system harmonics / J. Arrillaga, N.R. Watson. - $2^{\text {nd }}$ edit. - Chichester: Wiley, 2003.

[6] Voropai N.I., Kovernikova L.I., Molin N.I., Smirnov S.S. Analysis and normalization of harmonic conditions in $220 \mathrm{kV}$ network feeding traction load. Proceedings of international conference on Electrical Engineering,Vol.2, Xi'an, China, July 22-26, 2001, pp.960-963.

[7] Zhezhelenko I.V. High harmonics in the power supply systems of industrial plants. The 4th edition. Moskva: Energoatomizdat, 2000. 331 p. (Russia) 